\title{
DIRECT DECOMPOSITION OF REGULAR SEMIGROUPS
}

\section{R. J. WARNE}

1. Introduction. If $X$ is a semigroup, let $E_{X}$ denote the set of idempotents of $X$. The major purpose of this paper is to show that $S$ is a regular semigroup with $E_{S} \cong K \times B$, where $K$ is a semilattice and $B$ is a rectangular band, if and only if $S \cong T \times B$ where $T$ is an inverse semigroup with $E_{T} \cong K$. In the case $S$ is bisimple, the structure of $S$ may be described completely mod groups for several classes of semilattices $K$ (Remark 1). In the case $S$ is a Clifford semigroup, its structure may also be completely determined (Remark 2). A characterization of certain classes of regular semigroups, including right groups, is an immediate consequence of the theorem.

We adopt the terminology and notation of [1].

\section{The decomposition theorem.}

THEOREM. $S$ is a regular semigroup such that $E_{S} \cong K \times B$ where $K$ is a semilattice and $B$ is a rectangular band if and only if $S \cong T \times B$ where $T$ is an inverse semigroup with $E_{T} \cong K$. $S$ is bisimple if and only if $T$ is bisimple.

Proof. Let $S$ be a regular semigroup such that $E_{S} \cong K \times B$ where $K$ is a semilattice and $B$ is a rectangular band. Let $B$ be the set product $I \times J$ under the multiplication $(i, j)(k, s)=(i, s)$. If $a \in S, a \in R_{(e, i, j)}$ $\cap L_{(f, k, s)}$ where $(e, i, j),(f, k, s) \in K \times B$ since $S$ is a regular semigroup. Thus, $a=(e, i, j) a=a(f, k, s)$. Hence, $(g, r, t) a=(g, r, q) a$ and $a(g, p, q)$ $=a(g, r, q)$ for $g \in K, r, p \in I$ and $t, q \in J$. (We will use this fact several times in the proof without further mention.) Therefore, $a \in(e, i, s)$ $\cdot S(f, i, s)$ and

$$
S=\bigcup\{(e, i, j) S(f, i, j): e, f \in K, i \in I, j \in J\} .
$$

Let $i_{0}$ and $j_{0}$ be fixed elements of $I$ and $J$ respectively. (The elements $i_{0}$ and $j_{0}$ may be selected arbitrarily. Then, they are fixed.)

We will define an isomorphism $\theta$ of

$$
M=\left(\cup\left\{\left(e, i_{0}, j_{0}\right) S\left(f, i_{0}, j_{0}\right): e, f \in K\right\}\right) \times B
$$

onto $S=\bigcup\{(e, i, j) S(f, i, j): e, f \in K, i \in I, j \in J\}$ in the following manner:

$(x, i, j) \theta=(e, i, j) x(f, i, j) \quad$ where $\quad(x, i, j) \in\left(\left(e, i_{0}, j_{0}\right) S\left(f, i_{0}, j_{0}\right)\right) \times B$.

Presented to the Society, April 13, 1968; received by the editors June 10, 1966. 
First, we show that $\theta$ is single valued. Let $u=v=(x, i, j) \in\left(\left(\left(e, i_{0}, j_{0}\right)\right.\right.$ $\left.\left.S\left(f, i_{0}, j_{0}\right)\right) \times B\right) \cap\left(\left(\left(g, i_{0}, j_{0}\right) S\left(h, i_{0}, j_{0}\right)\right) \times B\right)$.

Thus,

$$
\begin{aligned}
(e, i, j) x(f, i, j) & =(e, i, j)\left(\left(g, i_{0}, j_{0}\right) x\left(h, i_{0}, j_{0}\right)\right)(f, i, j) \\
& =\left(e g, i, j_{0}\right) x\left(h f, i_{0}, j\right) \\
& =(g, i, j)\left(\left(e, i_{0}, j_{0}\right) x\left(f, i_{0}, j_{0}\right)\right)(h, i, j) \\
& =(g, i, j) x(h, i, j) .
\end{aligned}
$$

Hence $u \theta=v \theta$.

Next, we show that $\theta$ is one-to-one. Suppose that $u \theta=v \theta$ where $u \in\left(e, i_{0}, j_{0}\right) S\left(f, i_{0}, j_{0}\right) \times B$ and $v \in\left(g, i_{0}, j_{0}\right) S\left(h, i_{0}, j_{0}\right) \times B$. Let $u$ $=(x, i, j)$ and $v=(y, k, s)$. Thus, $(e, i, j) x(f, i, j)=(g, k, s) y(h, k, s)=z$, say. Hence, $z=(e, i, j) z=(g, k, s) z$. Since $S$ is a regular semigroup, there exists $z^{\prime} \in S$ such that $z z^{\prime} \in E_{S}$. Thus, suppose that $z z^{\prime}=(t, p, q)$ where $t \in K, p \in I$, and $q \in J$. Hence, $(e, i, j)(t, p, q)=(g, k, s)\left(t, p,{ }^{k} q\right)$, $(e t, i, q)=(g t, k, q)$, and $i=k$. In a similar manner $j=s$. Thus,

$$
(e, i, j) x(f, i, j)=(g, i, j) y(h, i, j)=\left(g, i, j_{0}\right) y\left(h, i_{0}, j\right) .
$$

Thus,

$$
\begin{aligned}
\left(g, i_{0}, j_{0}\right)\left(g, i, j_{0}\right) y\left(h, i_{0}, j\right)\left(h, i_{0}, j_{0}\right) & =\left(g, i_{0}, j_{0}\right)(e, i, j) x(f, i, j)\left(h, i_{0}, j_{0}\right), \\
\left(g, i_{0}, j_{0}\right) y\left(h, i_{0}, j_{0}\right) & =\left(g e, i_{0}, j\right) x\left(f h, i, j_{0}\right), \\
y & =\left(e, i_{0}, j_{0}\right)\left(g, i_{0}, j\right) x\left(h, i, j_{0}\right)\left(f, i_{0}, j_{0}\right) .
\end{aligned}
$$

Hence, $(y, k, s) \in\left(e, i_{0}, j_{0}\right) S\left(f, i_{0}, j_{0}\right) \times B$. Thus

$$
\begin{aligned}
(e, i, j) x(f, i, j) & =(e, i, j) y(f, i, j), \\
(e, i, j)\left(x\left(f, i_{0}, j_{0}\right)\right)(f, i, j)\left(f, i_{0}, j_{0}\right) & =(e, i, j)\left(y\left(f, i_{0}, j_{0}\right)\right)(f, i, j)\left(f, i_{0}, j_{0}\right), \\
(e, i, j) x\left(f, i_{0}, j_{0}\right) & =(e, i, j) y\left(f, i_{0}, j_{0}\right), \\
(e, i, j) x & =(e, i, j) y \\
\left(e, i_{0}, j_{0}\right)(e, i, j)\left(\left(e, i_{0}, j_{0}\right) x\right) & =\left(e, i_{0}, j_{0}\right)(e, i, j)\left(\left(e, i_{0}, j_{0}\right) y\right), \\
\left(e, i_{0}, j_{0}\right) x & =\left(e, i_{0}, j_{0}\right) y \\
x & =y
\end{aligned}
$$

Hence, $u=v$ and $\theta$ is one-to-one.

Next, we show that $\theta$ maps $M$ onto $S$. Let $(e, i, j) x(f, i, j) \in S$. Thus,

$$
\begin{aligned}
\left(\left(e, i_{0}, j_{0}\right) x\left(f, i_{0}, j_{0}\right), i, j\right) \theta & =(e, i, j)\left(\left(e, i_{0}, j_{0}\right) x\left(f, i_{0}, j_{0}\right)\right)(f, i, j) \\
& =\left(e, i, j_{0}\right) x\left(f, i_{0}, j\right) \\
& =(e, i, j) x(f, i, j) .
\end{aligned}
$$


Finally, we show that $\theta$ is a homomorphism. Let $(x, i, j) \in\left(e, i_{0}, j_{0}\right)$ $\cdot S\left(f, i_{0}, j_{0}\right) \times B$ and $(y, k, s) \in\left(g, i_{0}, j_{0}\right) S\left(h, i_{0}, j_{0}\right) \times B$. Hence,

$$
\begin{aligned}
(x, i, j) \theta(y, k, s) \theta & =(e, i, j) x(f, i, j)(g, k, s) y(h, k, s) \\
& =(e, i, s) x(f, i, j)(g, k, s) y(h, i, s) \\
& =(e, i, s)\left(x\left(f, i_{0}, j_{0}\right)\right)(f, i, j)(g, k, s)\left(\left(g, i_{0}, j_{0}\right) y\right)(h, i, s) \\
& =(e, i, s) x\left(f g, i_{0}, j_{0}\right) y(h, i, s) \\
& =(e, i, s) x\left(f, i_{0}, j_{0}\right)\left(g, i_{0}, j_{0}\right) y(h, i, s) \\
& =(e, i, s) x y(h, i, s) .
\end{aligned}
$$

Since $x y \in\left(e, i_{0}, j_{0}\right) S\left(f, i_{0}, j_{0}\right)\left(g, i_{0}, j_{0}\right) S\left(h, i_{0}, j_{0}\right) \subseteq\left(e, i_{0}, j_{0}\right) S\left(h, i_{0} j_{0}\right)$, $((x, i, j)(y, k, s)) \theta=(x y, i, s) \theta=(e, i, s) x y(h, i, s)$. Thus, $((x, i, j)(y, k, s)) \theta$ $=(x, i, j) \theta(y, k, s) \theta$.

Let $T=\bigcup\left\{\left(e, i_{0}, j_{0}\right) S\left(f, i_{0}, j_{0}\right): e, f \in K\right\}$. Hence, we have shown that $S \cong T \times B$. Clearly, $T$ is a semigroup with $E_{T}=\left\{\left(e, i_{0}, j_{0}\right): e \in K\right\} \cong K$. If $a \in\left(e, i_{0}, j_{0}\right) S\left(f, i_{0}, j_{0}\right)$ there exists $x \in S$ such that $a=a x a=$ $a\left(\left(f, i_{0}, j_{0}\right) x\left(e, i_{0}, j_{0}\right)\right) a$ and, hence, $T$ is regular. By [1, Theorem 1.17], $T$ is an inverse semigroup. The converse follows by a routine calculation.

CoRollary. $S$ is a regular semigroup whose idempotents form a rectangular band $B$ if and only if $S \cong G \times B$ where $G$ is a group. In particular, $S$ is a regular semigroup whose idempotents form a right zero semigroup if and only if $S$ is a right group.

In the next two remarks, we use the notation of the theorem.

REMARK 1. The theorem is of particular interest in the case $S$ is bisimple. In this case, $T$ has been described completely mod groups for several classes of $K$ (for example, see [3] and [4]). Hence, in these cases, the structure of $S$ may be described completely mod groups.

REMARK 2. We now give another case of particular interest. A semigroup which is a union of groups is called a Clifford semigroup [2, p. 43]. Clearly, a Clifford semigroup is regular. $S$ is a Clifford semigroup if and only if $T$ is a Clifford semigroup [1, p. 130, Exercise 10]. Hence, since in this case the structure of $T$ is known completely mod groups $[1$, p. 128, Theorem 4.11], the structure of $S$ is also known.

REMARK 3. The theorem represents a natural initial step in the study of the structure of regular semigroups $S$ such that $E_{S}$ is a subsemigroup beyond the inverse semigroups. We also take a step in the solution of the following problem: If $S$ is a regular semigroup such that $E_{S}$ is a subsemigroup, how is the structure of $S$ affected by that of $E_{S}$ ? 


\section{BIBLIOGRAPHY}

1. A. H. Clifford and G. B. Preston, The algebraic theory of semigroups, Math. Surveys, No. 7, Vol. 1, Amer. Math. Soc., Providence, R. I., 1961.

2. K. H. Hofmann and P. S. Mostert, Elements of compact semigroups, Merrill, Columbus, Ohio, 1966.

3. R. J. Warne, I-bisimple semigroups, Trans. Amer. Math. Soc. 130 (1968), $367-386$.

4. - Bisimple inverse semigroups mod groups, Duke Math. J. 34 (1967), 787-812.

WEST ViRgINIA UNIVERSITY 